Int. J. Morphol.,

31(3):997-1002, 2013

\title{
Método para la Generación de Modelos de Elementos Finitos de Dientes
}

\author{
Method to Generation of Finite Element Models of Teeth
}

Gisaku Kuramochi' ${ }^{*, * *} \&$ Mariano del Sol ${ }^{*, * * * *}$

KURAMOCHI, G. \& DEL SOL, M. Método para la generación de modelos de elementos finitos de dientes. Int. J. Morphol., 31(3):997$1002,2013$.

RESUMEN: El objetivo del estudio fue realizar una modelación 3D con la características morfológicas de un primer premolar superior a partir de su morfometría. Teniendo como referencia un diagrama anatómico de un primer premolar superior y sus variables morfométricas, se realizó el trazado de su contorno (software Catya) considerando al menos tres cortes transversales de la figura: vista oclusal, mesio-distal (AP) y uno vestíbulo-palatino (L). El modelo 2 d obtenido se completó mediante extrapolación para obtener una imagen tridimensional en malla que, posteriormente, se transforma a elementos triangulares, quedando el modelo de premolar listo para ser importado al software de análisis de elementos finitos (Ansys). Aquí, estos elementos son transformados a su vez en elementos placa, unidos por haces, lo que le confiere un comportamiento lineal e isométrico. A éstos, se ingresan los valores de las propiedades mecánicas correspondiente a cada tejido para proceder al análisis. La figura así obtenida, permite reproducir la morfología del premolar y las relaciones entre sus componentes. Además, la linealidad e isometría conferida permite estudiar el comportamiento mecánico en cualquier sección del modelo. El diseño computacional de estructuras anatómicas, a partir de un dibujo de contorno en base a su morfología y morfometría con la técnica descrita, resulta un alternativa complementaria para estudiar la morfología y realizar análisis mecánicos de dientes u otras estructuras anatómicas.

PALABRAS CLAVE: Morfología, Morfometría, Analisis de Elementos Finito, rasgos morfológicos.

\section{INTRODUCCIÓN}

Es un hecho conocido que el desarrollo de distintos softwares de modelación asistida por computador (CAM) permiten la generación de imágenes de distintas estructuras en 2D y 3D notables, con una excelente reproductibilidad de detalles. De igual manera, se sabe que el costo de estas herramientas computacionales es elevadísimo, sin embargo, la mayor dificultad estriba en el conocimiento y entrenamiento necesarios para su manejo y utilización en un área específica (Vasudeva, 2009), lo que de alguna manera ha llevado a una subutilización de ellas en distintas áreas del conocimiento.

Para la morfología, como parte de la biología, que estudia la forma de los seres orgánicos y de las modificaciones o transformaciones que experimenta, resulta especialmente interesante disponer de elementos capaces de realizar modelaciones 2 y 3D. De igual forma lo es para la Odontoscopía o morfología dental cuyo objeto es observar, registrar, analizar y comprender el comportamiento de la expresión (frecuencia y variabilidad) de la morfología coronal y radicular de los dientes humanos (Rodríguez, 2005). La morfología de los dientes está determinada por una serie de rasgos morfológicos dentales coronales y radiculares, como resultado de la expresión fenotípica del genoma de un individuo o una población (Girón, 2009). Estos rasgos se caracterizan por su frecuencia, variabilidad, bilateralidad, dimorfismo sexual, correspondencia y valor taxonómico, cuyo registro, observación y análisis científico resultan ser relevantes para distintas disciplinas (Rodríguez, 1989; Rodríguez, 2003).

Por su parte la odontometría o la obtención de las medidas coronales y radiculares de los dientes, son utilizadas para distintos fines de acuerdo al campo de estudio, en Antropología para estudios evolutivos y relaciones filogenéticas, en Ortodoncia para predecir el espacio o in-

* Programa de Doctorado en Ciencias Médicas, Universidad de La Frontera, Temuco, Chile.

** Universidad Finis Terrae, Santiago, Chile.

*** Centro de Investigación en Ciencias Biomédicas, Universidad Autónoma de Chile, Chile.

Proyecto de Tesis de Doctorado en Ciencias Médicas, Universidad de La Frontera, Chile. 
cluso en el área forense para determinar el sexo de un individuo (Rodríguez, 1989, 2004; Scott \& Turner, 1998; Zoubov, 1998). Debido a la ubicación anatómica de los puntos de referencia, éstos no están sujetos a variaciones por acción de agentes mecánicos externos (Rodríguez, 2003), por lo tanto las medidas odontométricas de mayor objeto de estudio son el diámetro mesio-distal, definido como la distancia entre los puntos interproximales mesial y distal de mayor contorno y el diámetro vestíbulo-palatino (lingual en el caso de los dientes inferiores), definido como la distancia entre las máximas convexidades de las superficies vestibular y palatina (lingual) (Rodríguez, 1989; Bernabé et al., 2005).

Sin embargo, a pesar de esta aparente simplificación, un aspecto que resulta no menor cuando se pretende modelar una estructura, es la cantidad de tiempo requerido para el diseño y obtención de la imagen volumétrica necesaria para la realización de los análisis; esta etapa a su vez es la más crítica debido a que en la medida que reproduce de manera más fiel la estructura a estudiar permitirá una mayor precisión del análisis.

Se han desarrollado distintas técnicas de adquisición de imágenes (Magnet, 2006) con un nivel de fidelidad cercano al $99 \%$, sin embargo se requiere de distintos softwares manejados por expertos para llegar a la modelación final, lo que encarece cuando no alarga el proceso.

Así entonces para poder comparar y apreciar aquellos elementos morfológicos que den cuenta de cambios para distintas disciplinas (biología, antropología, odontología, paleopatología, arqueología, ciencias forenses, entre otras), nuestro objetivo consistió en proponer una técnica que constituya una alternativa simplificada para adquirir imágenes y diseñar mediante un software una modelación volumétrica 3D con los rasgos morfológicos de un primer premolar superior a partir de su morfometría.

\section{MATERIAL Y MÉTODO}

\section{Proceso de reconstruccción 3D de un primer premolar superior.}

1.- A partir de las figuras obtenidas desde la fotografía (Fig. 1) y las dimensiones dadas (Tabla I) se elaboran curvas de nivel primario, que delinean los contornos de los planos principales del premolar con el Software Catia (Figs. 2 y 3).

2.- A partir de las secciones y las curvas de nivel se comienza un tejido de malla de líneas sobre la superficie exterior (teórica) del diente. (Figs. 4, 5 y 6).
Tabla I. Valores morfométricos promedios de un primer premolar superior según Figún \& Garino, 2002.

\begin{tabular}{lll} 
Longitud del diente & 21 & $\mathrm{~mm}$ \\
Longitud cavidad pulpar & 17,8 & $\mathrm{~mm}$ \\
Diámetro coronario vestibulopala tino & 9 & $\mathrm{~mm}$ \\
Espesor de la pared vestibular & 3,2 & $\mathrm{~mm}$ \\
Ancho vestíbulopalatino de la cámara & 4 & $\mathrm{~mm}$ \\
Espesor de la pared palatina & 2,5 & $\mathrm{~mm}$ \\
Diámetro coronario mesiodistal & 7 & $\mathrm{~mm}$ \\
Espesor de la pared mesial & 2 & $\mathrm{~mm}$ \\
Ancho mesiodistal de la cámara & 3 & $\mathrm{~mm}$ \\
Espesor de la pared distal & 2 & $\mathrm{~mm}$ \\
\hline
\end{tabular}
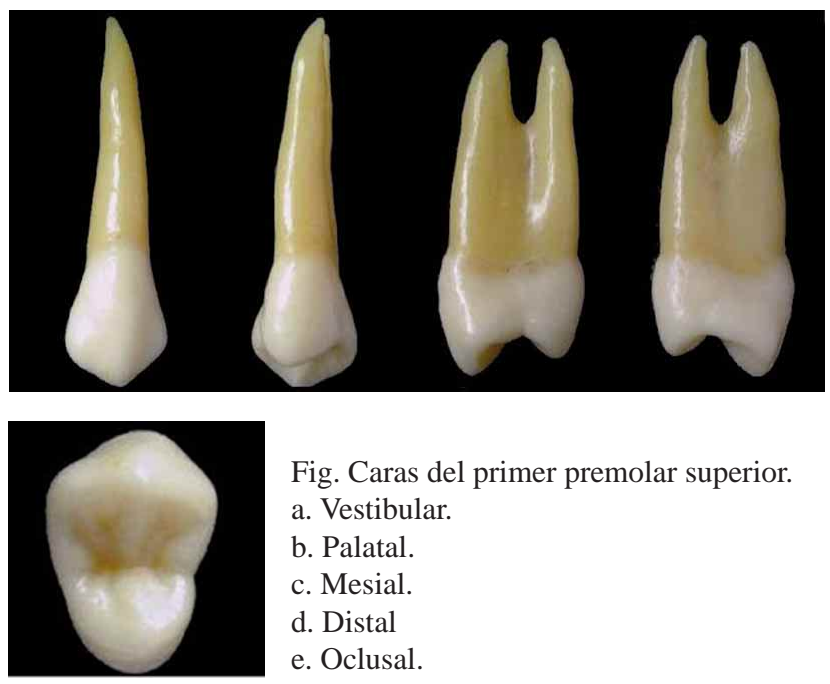

Fig. Caras del primer premolar superior. a. Vestibular. b. Palatal. c. Mesial. d. Distal e. Oclusal.

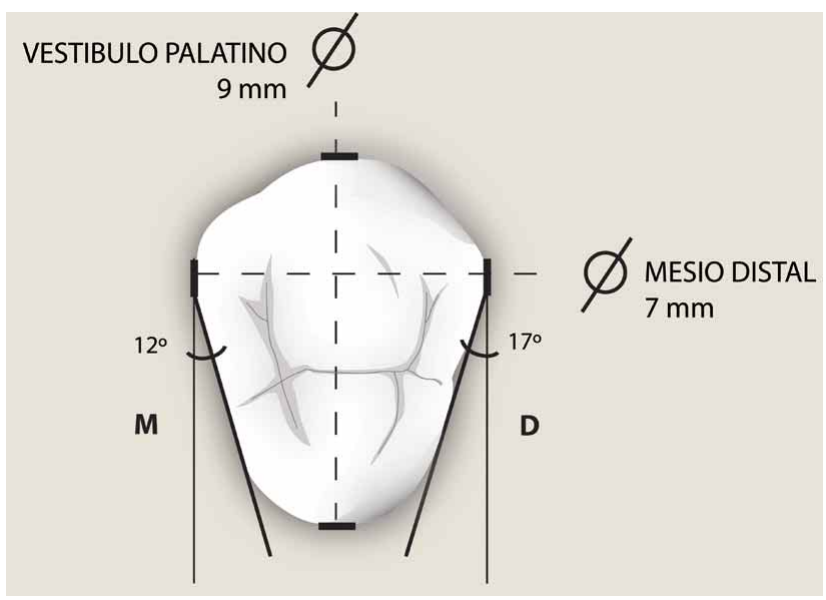

Fig. 2. Dimensiones del diente.Vista oclusal del primer premolar superior. (Valores morfométricos).

3.- Se repite el proceso dobteniéndose 3 capas concéntricas, separadas aproximadamente $1 \mathrm{~mm}$ una de la otra. Se obtiene un modelo con 3 superficies; una exterior, una intermedia y una interior. Como resultado se modela también el vaciado interior del premolar (Figs. 7 y 8). 


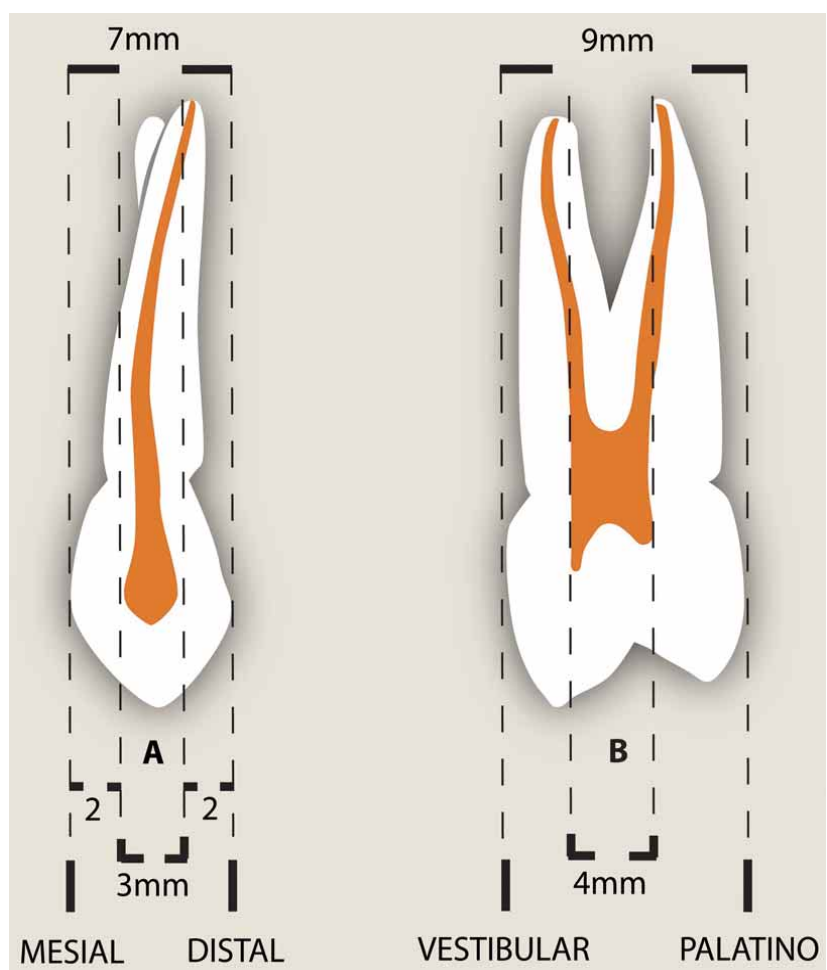

Fig. 3. Dimensiones del diente. Vista/ sección/corte vestíbulopalatina y mesiodistal.

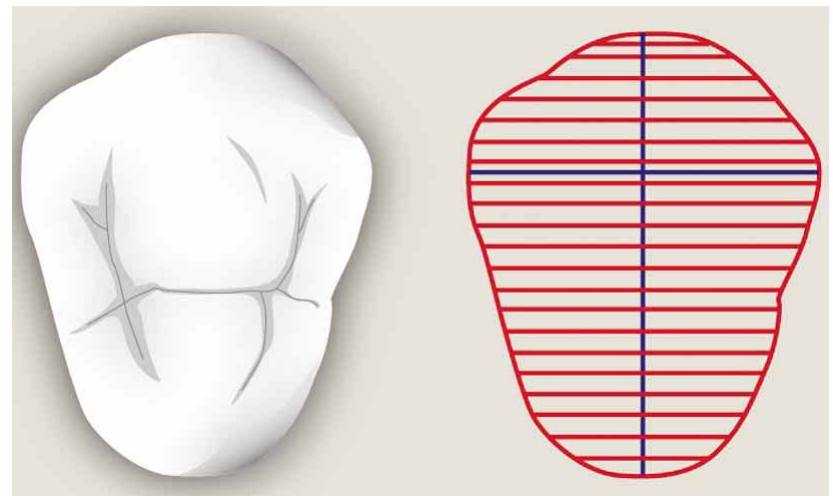

Fig. 4. Vista oclusal del primer premolar superior. Tejido de malla de líneas sobre la superficie exterior.

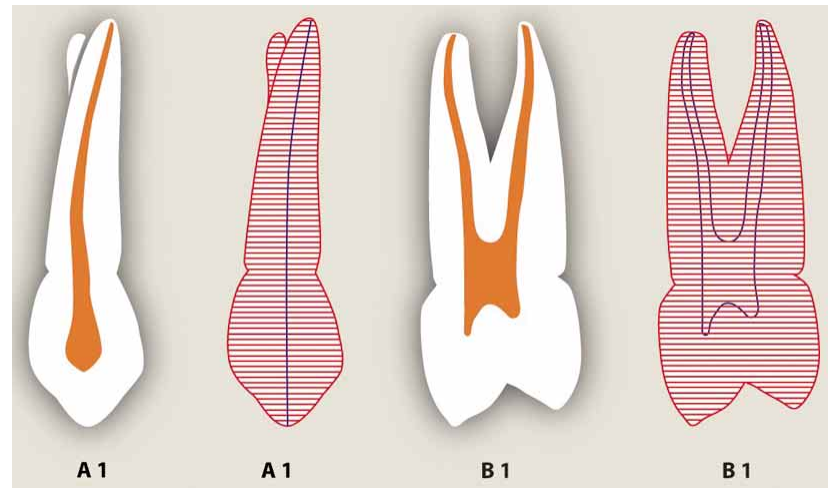

Fig. 5. Vista/ sección/corte vestíbulopalatina y mesiodistal.Tejido de malla de líneas sobre la superficie exterior.

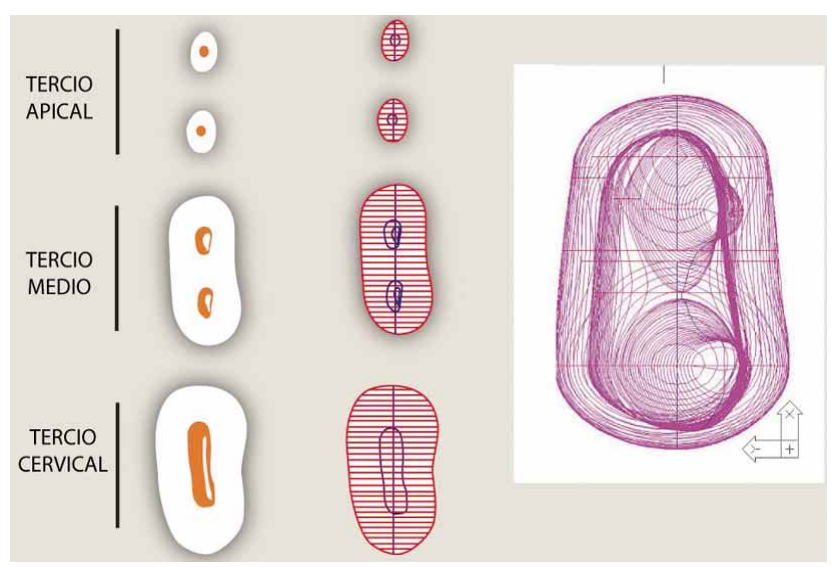

Fig. 6. Secciones a nivel radicular. Tejido de malla de líneas sobre la superficie exterior.

4.- Se elaboró un modelo computacional del premolar mediante el software Catya, versión 8.1 del año 2009. El modelo consta de 13715 nodos y 29937 elementos placa; estructurándose mediante 3 capas de dentina a nivel radicular:

Una capa exterior de 1 [mm] de espesor. La geometría de esta capa corresponde a la morfología exterior del diente.

Una capa media de $0.5[\mathrm{~mm}]$ de espesor. En esta capa se une la capa exterior con la interior.

Una capa interior de $1[\mathrm{~mm}]$ de espesor. Esta capa representa la pared interior del premolar.

Estas capas se unen por nervios longitudinales de dentina (Fig. 9), de modo de formar una estructura rígida sólida y homogénea que modela el espesor total de la dentina.De esta manera, se obtiene un modelo isotrópico, elástico y homogéneo, que permite realizar a posterior simulaciones de pruebas mecánicas.

\section{DISCUSIÓN}

Esta técnica puede ser un aporte interesante para la investigación, con el que se consigue una ahorro importante de tiempo y de recursos. Esta situación permitiría acceder a este tipo de herramientas computacionales con mayor facilidad permitiendo generar nuevas estrategias para estudios morfológicos interdisciplinarios.

Así también es destacable la posibilidad que ofrece de variar la dimensiones del modelo de la estructura anatómica a estudiar, permitiendo por consiguiente comparar una 


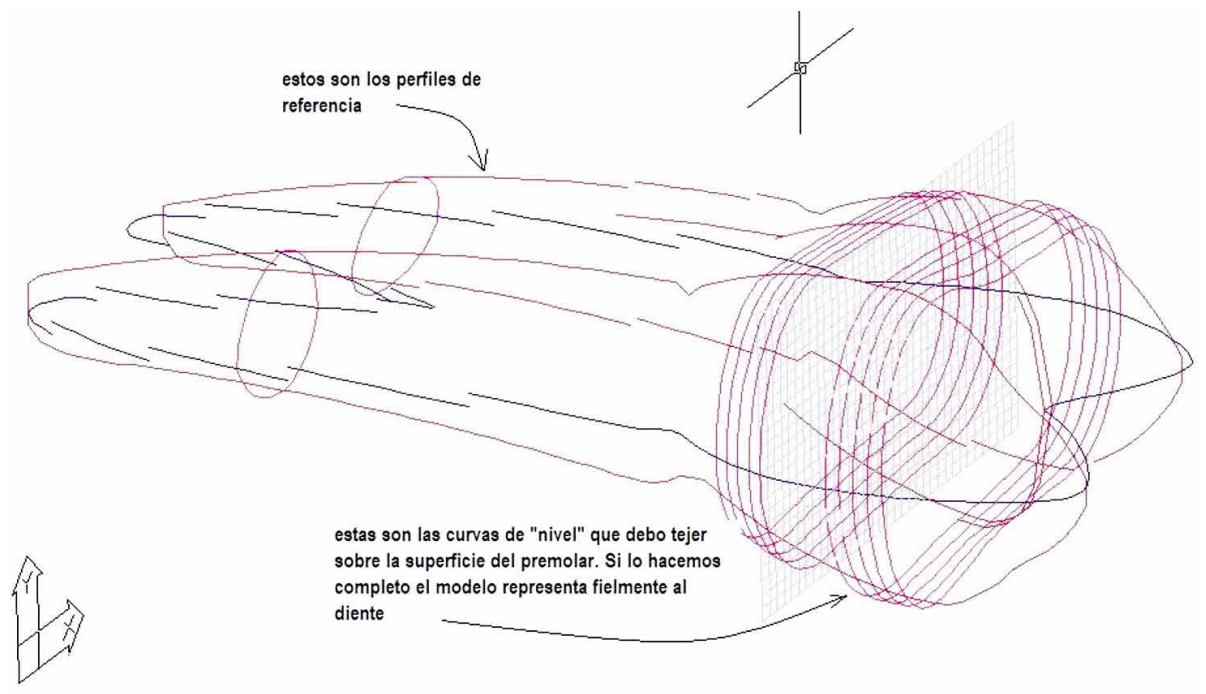

Fig. 7. Malla sobre curvas de nivel.

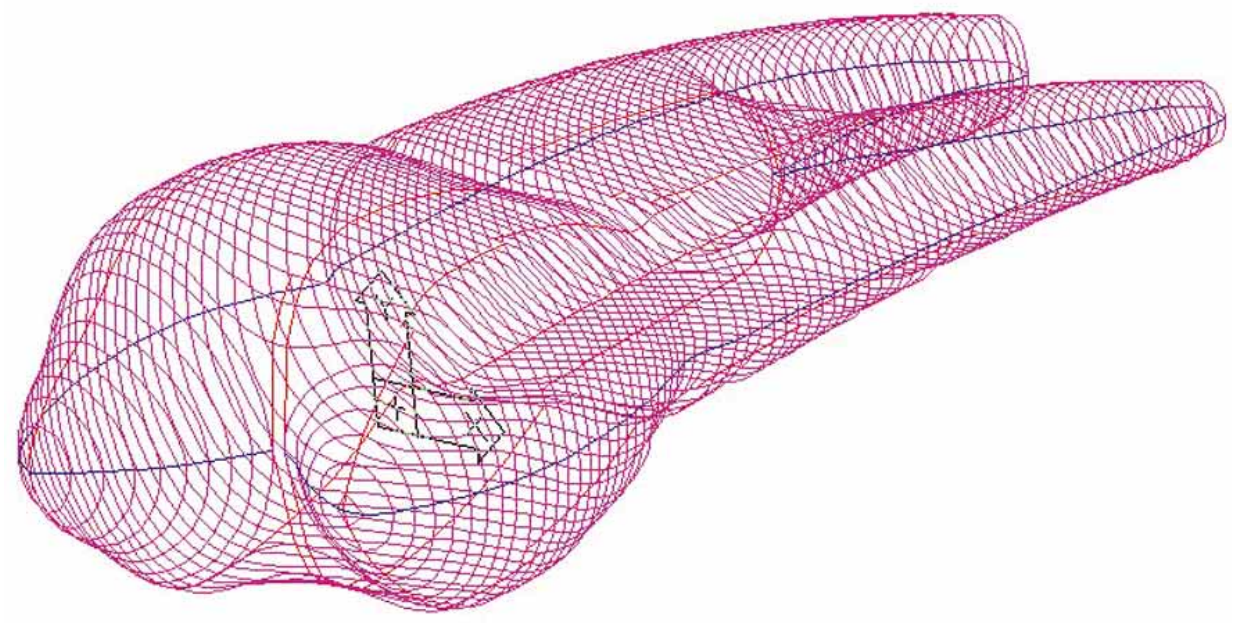

Fig. 8. Diseño de la malla sobre curvas de nivel.

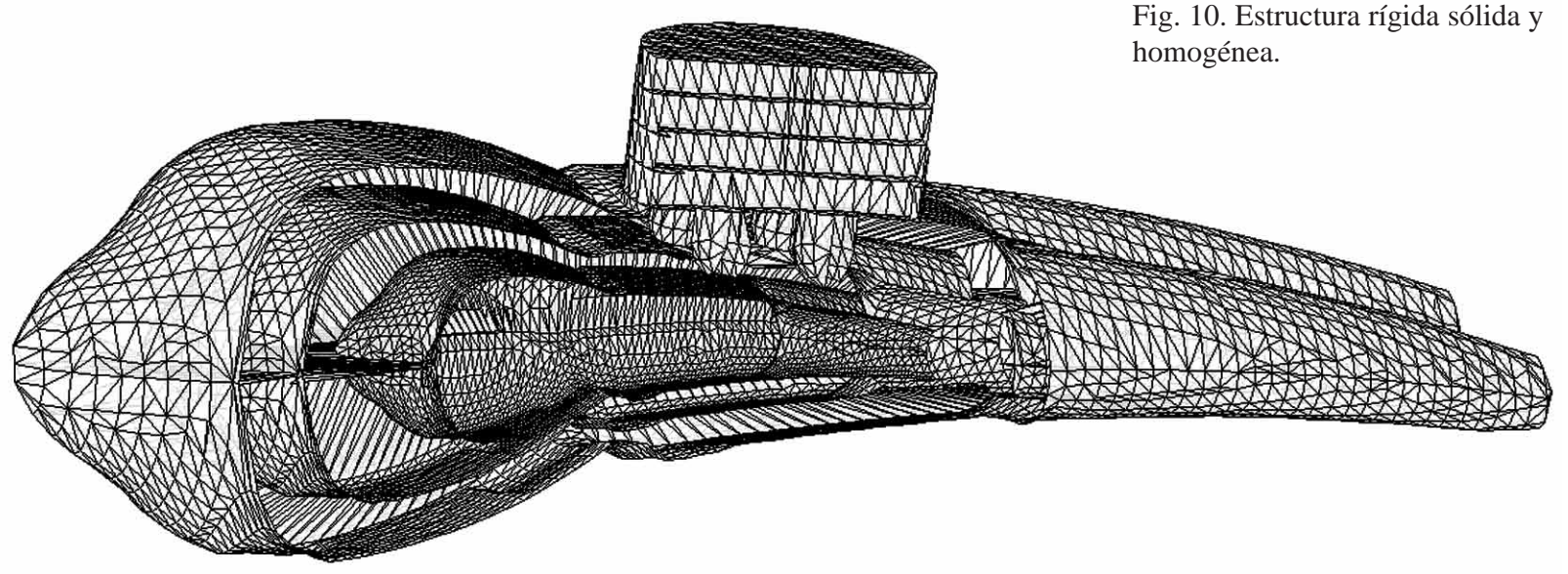

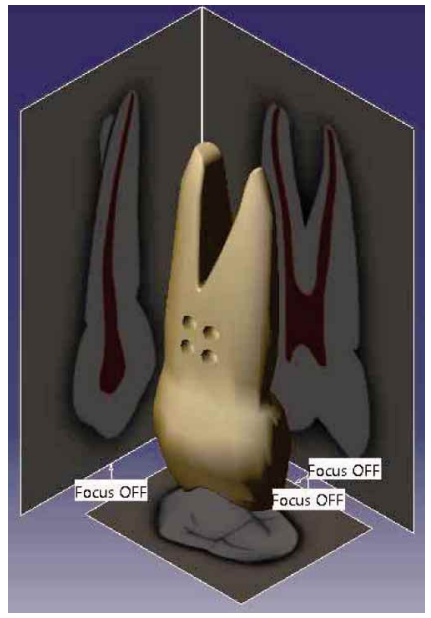

Fig. 10. Estructura rígida sólida y homogénea.

Fig. 9. Modelo 3D final del diente. Se encuentra incluído cilindro. 
misma figura a partir de otras relaciones morfométricas sin perder lo realizado (Figún \& Garino, 2002; Fuentes et al., 2011).

Generalmente en los modelos 3D la reproducción fina de los rasgos morfológicos dentales coronales y radiculares está limitada a dos factores; al método de adquisición de la geometría (trazado manual, micro CT) y a la capacidad de memoria que demanda la modelización (Magnet). En consecuencia a pesar de la versatilidad de esta técnica debemos asumir sus limitaciones debido a que para la obtención de nuestro modelo en su etapa de diseño fue necesario extrapolar.

La necesidad de obtener modelos 3D con una alta reproductibilidad de detalles y precisión no solo del contorno sino también de la geometría interna ha devenido en la búsqueda de variadas aproximaciones utilizando distintas metodologías (Verdonchot et al., 2001), son quizás los primeros en desarrollar un modelo 3D a partir de la adquisición de datos con una técnica Micro CT. Una propuesta semiautomatizada bastante interesante fue la desarrollada por Cattaneo et al. (2001), que para obtener un modelo sólido de hueso utilizaron las unidades Hounsfield (HU) de densidad de escaneado, sin embargo, no era posible tener un control fino de los límites internos en estructuras peque- ñas como los dientes (línea amelo-dentinaria). Este problema fue solucionado por un estudio (Magne) que muestra que la obtención máxima de detalles anatómicos se adquiere con un mallaje basado en una relación superficie/interfase utilizando Scanner y esterereolitrografía obteniendo de manera más rápida un modelo 3D para Fem, el que además fue validado.

A pesar de estos avances, los requerimientos de especialistas altamente capacitados es aún insoslayable. Por lo tanto se hace necesario potenciar el acceso a estas tecnologías /softwares mediante el desarrollo de herrramientas de modelación matemáticas más simples lo que nos ayudaría a comparar y así apreciar aquellos elementos morfológicos que den cuenta de cambios para distintas disciplinas biología, antropología, odontología, paleopatología, arqueología y ciencias forenses.

Finalmente, de acuerdo con la estructuración realizada para el modelo de premolar, se puede concluir que él representará su morfología y comportamiento mecánico entregando información confiable sobre los esfuerzos y deformaciones en la zonas en estudio que se requieran. Por lo tanto esta técnica puede ser utilizado para el diseño y estudio de distintas estructuras anatómicas.

KURAMOCHI, G. \& DEL SOL, M. Method to generation of finite element models of teeth. Int. J. Morphol., 31(3):997-1002, 2013.

SUMMARY: The aim of this study was making a 3D modeling with the morphological characteristics of a first premolar from its morphometry. With reference to an anatomical diagram of a first upper premolar and morphometric relationships, it performs its contour plot (software Catya) considering at least three cross-sections of the figure seen occlusal, mesial-distal (AP) and one vestibulopalatal (L). $2 \mathrm{~d}$ obtained model is completed by extrapolation to obtain a three-dimensional mesh which subsequently transforms triangular elements premolar model being ready to be imported into the software of the finite element analysis (Ansys) where these elements are transformed into the once plate elements connected by beams which gives a linear and isometric to which also inputs values ??of mechanical properties for each tissue for the analysis. Thus the figure obtained allows us to reproduce the morphology of the premolar and the relationships among its components, including linearity and isometry conferred allows us to study the mechanical behavior in any section of the model. Computational design of anatomical structures from a contour drawing based on their morphology and morphometry using the technique described results in a complementary alternative to study the morphology and mechanical analyzes of teeth or other anatomical structures.

KEYWORDS: Morphometry; Finite Element Analysis; Dental morphology; Non-metric dental crown traits; Metric dental crown traits;

\section{REFERENCIAS BIBLIOGRÁFICAS}

Bernabé, E.; Lagravére, M. O. \& Flórez, C. Permanent dentition mesio-distal and bucco-lingual crown diameters in a Peruvian sample. Int. J. Dental Anthropol., 6:1-13, 2005.

Cattaneo, P. M.; Dalstra, M. \& Frich, L. H. A three-dimensional finite element model from computed tomography data: a semiautomated method. Proc. Inst. Mech. Eng. [H];215:203131961;40:936-45, 2001.
Figún, M. E. \& Garino, R. R. Anatomía odontológica: funcional y aplicada. 2. Ed. Buenos Aires, El Ateneo, 2002. pp. 234-40,

Fuentes, F. R.; Borie E. E.; Bustos, M. L. \& Tomas, M. D. First Premolar Morphometry: A Study in 54 Cases. Int. J. Morphol., 29(1):234-9, 2011.

Girón, G.; Gómez, P.; Morales, L.; León, M. \& Moreno, F. Rasgos 
morfológicos y métricos dentales coronales de premolares superiores e inferiores en escolares de tres instituciones educativas de Cali, Colombia. Int. J. Morphol., 27(3):913-25, 2009.

Magne, P. Efficient 3D finite element analysis of dental restorative procedures using micro-CT data. Dent. Mater., 23:539-48, 2007.

Rodríguez, C. D. La antropología dental y su importancia en el estudio de los grupos humanos. Rev. Fac. Odont. Univ. Ant., 16(1-2):52-9, 2005.

Rodríguez, J. V. Introducción a la antropología dental. Cuad. Antrop., 19:1-41, 1989.

Rodríguez, J. V. Dientes y diversidad humana: avances de la antropología dental. Santa Fe de Bogotá, Universidad Nacional de Colombia, 2003.

Rodríguez, J. V. La antropología forense en la identificación humana. Santa Fe de Bogotá, Universidad Nacional de Colombia, 2004.

Scott, G. C. \& Turner, II C. G. Dental anthropology. Ann. Rev. Antrophol., 17:99-126, 1998.

Vasudeva G. Finite element analysis: A Boon To Dental Research. The Internet Journal of Dental Science. Vol. 6. N ${ }^{\circ} 2$. DOI: 10.5580/2626, 2009.

Verdonschot, N.; Fennis, W. M.; Kuijs, R. H.; Stolk, J.; Kreulen, C. M. \& Creugers, N. H. Generation of 3D finite element models of restored human teeth using micro-CT techniques. Int. J. Prosthodont., 14:310-5, 2001.

Zoubov, A. A. La antropología dental y la práctica forense. Maguaré, 13:243-52, 1998.

\author{
Dirección para Correspondencia: \\ Gisaku Kuramochi \\ Departamento de Odontología Restauradora \\ Facultad de Odontología \\ Universidad Finis Terrae \\ Santiago - CHILE \\ Gisaku Kuramochi \\ Aldunate 620 \\ Temuco - CHILE \\ Teléfono: 56-45-2211882 \\ Email: gkuramochi@gmail.com
}

